\title{
Assessment of Knowledge on Signs and Symptoms of Menopause among Premenopausal Women
}

\author{
Prof.S.Kala Barathi ${ }^{1}$, Dr. S.Kalavathi ${ }^{2}$ \\ ${ }^{1}$ Saveetha College Of Nursing Saveetha University,India \\ ${ }^{2}$ Principal,RMCON, Annamalai University, India
}

\begin{abstract}
Menopause is a natural biologic process not an estrogen deficiency disease. It represents the permanent cessation of menses resulting from the loss of ovarian follicular function. Some may think of menopause as the "change of life". Many women will begin to experience the signs and symptoms of approaching menopause around 50 years of age. The objective of the study is to assess the level of knowledge regarding signs and symptoms of menopause among women in premenopausal period,and determine the association between the level of knowledge and demographic variables.A descriptive design was chosen to assess the level of knowledge on signs and symptoms of menopause among women in premenopausal period. Setting- selected hospital in Thiruvallur.Sampling Technique - convenient sampling technique.Sample Size - 30 samples.Major Findings Of The Study-Among 30 women 17(56\%) had inadequate knowledge, 11(37\%) had moderate adequate knowledge and only 2(7\%) had adequate knowledge about menopause.Conclusion-Many women feel that this is a subject that should not be discussed as it is embarrassing. However by keeping quiet, we may serve to frighten the women about what is happening to their bodies. So knowledge and awareness can be co-ordinated together to maintain complete health and fitness.
\end{abstract}

\section{Introduction}

Menopause is the permanent end of menstruation and fertility, defined as occurring 12 months after the last menstrual period. Menopause is a natural biological process, not a medical illness. Even so, the physical and emotional symptoms of menopause can disrupt your sleep, sap your energy and -at least indirectly- trigger of sadness and loss.

Menopause, the time when a woman stops having menstrual periods, is not a disease or an illness. It is a transition between two phases of a woman's life. Menopause is the time in a female's life when she gets a formal, official signal from her body that she is getting older. This is the time when her menstruation is on the verge of a complete die down. The hot flashes, irritation, mood swings, insomnia, fatigue, etc. are some symptoms of menopause, and there are many more. These symptoms can be unpleasant and tedious to manage

Many women experience a variety of symptoms as a result of the hormonal changes associated with the transition through menopause. Around the time of menopause, women often lose bone density and their blood cholesterol levels may worsen, increasing their risk of heart disease.

According to National Institute on Aging (2010) usually menopause happens naturally, but some women develop symptoms of menopause and stop having menstrual cycles much earlier than expected. Before age 40,a menopause-like condition can happen for no known reason, or it can be caused by radiation treatment, some medicines like those used in chemotherapy, an autoimmunity (some of a women's own body cells attacking her ovary or ovaries),or genetic errors. Radiation can make the ovaries stops workings, as can some treatments like chemotherapy for cancer.

\section{Need for the study}

Sharda sidhu,Avneet KaurandMandeepSidhu(2005)reported in their cross sectional study determined the median age at menopause and frequency of various related clinical symptoms among 539 educated womenbetween 40-50 years of Amritsar district of Punjab. (47.50\%) of the study population were classified as postmenopausal, i.e., having no vaginal bleeding during the last 12 months, and 283 women $(52.50 \%)$ were perimenopausal, i.e., having irregular vaginal bleeding during the last 12 months. Median age at menopause was $47.54 \pm 2.31$ years which is close to the estimates from other Punjabi populations. The most common clinical symptoms associated with menopause were hot flushes and night sweats(55.08\%), insomnia (53.12\%), headache and body-aches (38.28\%), fatigue (42.18\%), irritability (35.15\%), perspiration(34.76\%), palpitation (22.26\%), short breath $(20.31 \%)$, nervous tension $(10.56 \%)$ and depression $(8.20 \%)$.

Nusrat N,Nishat Z,Gulfareen H,Aftab M,Asia $\mathbf{N}(\mathbf{2 0 0 8})$ reported in their studythat among 863 women680(78.79\%)had inadequate knowledge,137(15.8\%)know the symptoms of menopause.Frequently reported symptoms were Backache in 653(75.66\%),Insomnia in544(63.44\%),vasomotor symptomsin 513(59.4\%)andnight sweat in 390(45.19\%).Loss of memory in536(62.10). 
Harbindar et al.,(2005)assed the prevalence of menopausal symptomsinMalaysia among 326women reduced concentration(77.5\%), musculoskeletal,aches,(70.6\%)and,backache(67.7\%), nightsweat(53\%)andhotflashes(44.8 $\%)$,modswings( $51 \%$ ), sleep problem(45.1\%), anxiety(39.8\%), urinarytract infection(19.3\%).

\section{Objectives:}

1. to assess the level of knowledge regarding signs and symptoms of menopause among women in premenopausal period,

2. to determine the association between the level of knowledge and demographic variables

\section{Assumption:}

1. Premenopausal women do not have adequate knowledge regarding menopause

2. Adequate knowledge on menopause will help the women to promote coping strategies during their menopausal period

\section{Research Design:}

A descriptive design was chosen to assess the level of knowledge on signs and symptoms of menopause among women in premenopausal period.

Setting- selected hospital in Thiruvallur.

Population- All women attending the OPD in the hospital

Sample- women in the age group of 35-45years

Sample size - 30 samples.

Sampling technique - convenient sampling technique.

\section{Criteria for sample selection:}

\section{Inclusion criteria}

1. Premenopausal women in $35-45$ years

2. Premenopausal who are willing to participate

3. Premenopausal who could speak English and Tamil

\section{Exclusion criteria}

1.Postmenopausal women

\section{Data Collection Procedure}

Permission was obtained from the Superintendent of the hospital.samples were selected using simple random sampling. The purpose of the study was explained and informed consent was obtained.The samples were made to seat comfortably and privacy was provided.The selected samples were interviewed,daily 3 to 4 samples were selectedfor the study.

\section{Data Analysis And Statistical Methods Used}

Data was collected, tabulated and analyzed using statistical methods mentioned in the following

TABLE.

\begin{tabular}{|c|l|l|l|}
\hline S.No & Data Analysis & Methods & Remarks \\
\hline 1 & DescriptiveStatistics & $\begin{array}{l}\text { Numbers,Percentage, } \\
\text { mean,Standard deviation }\end{array}$ & $\begin{array}{l}\text { To assess the level of knowledge in } \\
\text { Premenopausal women }\end{array}$ \\
\hline 2 & Inferential Statistics & Chisquare test & $\begin{array}{l}\text { To determine the association between the level of } \\
\text { knowledge and demographic characteristics of the } \\
\text { mothers }\end{array}$ \\
\hline
\end{tabular}




\section{Result}

Table 1Distribution of Demographic variables of the premenopausal women

\begin{tabular}{|c|c|c|c|}
\hline S.No & Demographic variables & No. & $\%$ \\
\hline $\mathbf{1}$ & $\begin{array}{cc}\text { Age in years: } \\
\begin{array}{cc}\text { a. } & 35-38 \\
\text { b. } & 39-42 \\
\text { c. } & 4345\end{array}\end{array}$ & $\begin{array}{c}9 \\
10 \\
11\end{array}$ & $\begin{array}{l}30 \\
33 \\
36\end{array}$ \\
\hline 2 & $\begin{array}{l}\text { Educational status: } \\
\begin{aligned} \text { a. } & \text { Non literate } \\
\text { b. } & \text { Primary } \\
\text { c. } & \text { High school } \\
\text { d. } & \text { Collegiate }\end{aligned}\end{array}$ & $\begin{array}{c}14 \\
7 \\
9\end{array}$ & $\begin{array}{l}40 \\
23 \\
30\end{array}$ \\
\hline 3 & 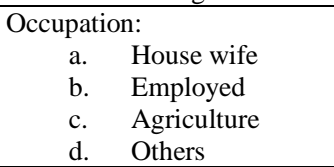 & $\begin{array}{c}19 \\
4 \\
7\end{array}$ & $\begin{array}{l}63 \\
13 \\
23\end{array}$ \\
\hline 4 & $\begin{array}{l}\text { Monthly lincome in Rupees: } \\
\begin{array}{cl}\text { a. } & \text { Below } 1000 \\
\text { b. } & 1001-2000 \\
\text { c. } & 2001-3000 \\
\text { d. } & \text { Above } 3000\end{array}\end{array}$ & $\begin{array}{c}13 \\
11 \\
5 \\
1\end{array}$ & $\begin{array}{c}43 \\
38 \\
16 \\
3\end{array}$ \\
\hline 5 & $\begin{array}{cl}\text { Religion: } & \\
\text { a. } & \text { Hindu } \\
\text { b. } & \text { Christian } \\
\text { c. } & \text { Muslim } \\
\text { d. } & \text { Others } \\
\end{array}$ & $\begin{array}{c}19 \\
10 \\
1\end{array}$ & $\begin{array}{c}63 \\
33 \\
3\end{array}$ \\
\hline 6 & $\begin{array}{cl}\text { Marital } & \text { Status: } \\
\text { a. } & \text { Single } \\
\text { b. } & \text { Married } \\
\text { c. } & \text { Widow } \\
\text { d. } & \text { Divorce } \\
\end{array}$ & $\begin{array}{c}1 \\
24 \\
5\end{array}$ & $\begin{array}{c}3 \\
80 \\
16\end{array}$ \\
\hline 7 & $\begin{aligned} \text { Parity: } & \\
\text { a. } & \text { Nullipara } \\
\text { b. } & \text { Primipara } \\
\text { c. } & \text { Multipara } \\
\text { d. } & \text { Grand multipara }\end{aligned}$ & $\begin{array}{c}2 \\
4 \\
16 \\
8\end{array}$ & $\begin{array}{c}6 \\
13 \\
53 \\
26\end{array}$ \\
\hline
\end{tabular}

Table 2 Distribution of over all level of knowledge among premenopausal women

\begin{tabular}{|c|l|c|c|}
\hline S.No & Different aspects & Mean & S.D. \\
\hline 1 & Meaning and its causes & 2.76 & 0.564 \\
\hline 2 & Signs and symptoms & 2.36 & 6.910 \\
\hline 3 & Coping strategies of menopause & 3.2 & 1.84 \\
\hline \multicolumn{2}{r|}{ Over all } & 8.6 & 2.5 \\
\hline
\end{tabular}

Table 3

\begin{tabular}{|c|l|c|c|}
\hline S.No & Level of knowledge & No & \% \\
\hline 1 & Inadequate & 17 & 56 \\
\hline 2 & Moderately adequate & 11 & 37 \\
\hline 3 & Adequate & 2 & 7 \\
\hline
\end{tabular}

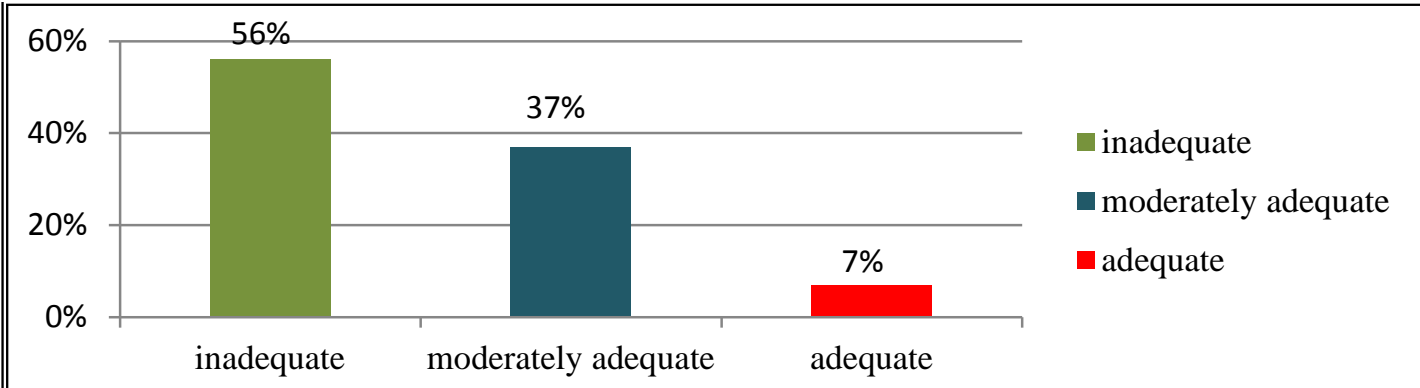

Figure: 1 Distribution of level of overall knowledge among premenopausal women 
Table 4Association between demographic variables and level of knowledge on menopase

\begin{tabular}{|c|c|c|c|c|c|c|c|c|}
\hline \multirow[t]{3}{*}{ S.No } & \multirow[t]{3}{*}{ Demographic variables } & \multicolumn{6}{|c|}{ Level of knowledge } & \multirow{3}{*}{$\begin{array}{l}\text { (P Value) } \\
\mathrm{X} 2 \text { value }\end{array}$} \\
\hline & & \multicolumn{2}{|c|}{$\begin{array}{l}<50 \\
\text { adequate }\end{array}$} & \multicolumn{2}{|c|}{$\begin{array}{l}\mathbf{5 0 - 7 0} \\
\text { Moderarely } \\
\text { adequate }\end{array}$} & \multicolumn{2}{|c|}{$\begin{array}{l}>70 \% \\
\text { adequate }\end{array}$} & \\
\hline & & No. & $\%$ & No. & $\%$ & No. & $\%$ & \\
\hline 1 & 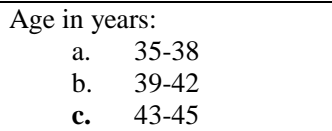 & $\begin{array}{l}7 \\
5 \\
5\end{array}$ & $\begin{array}{l}23 \\
16 \\
16\end{array}$ & $\begin{array}{l}2 \\
3 \\
6\end{array}$ & $\begin{array}{l}6.6 \\
10 \\
20\end{array}$ & $\begin{array}{l}0 \\
2 \\
0\end{array}$ & $\begin{array}{l}0 \\
6.6 \\
0\end{array}$ & $\begin{array}{c}\mathrm{X}^{2}=7.15 \\
\mathrm{Df}=4 \mathrm{NS} \\
\mathrm{P}<0.05\end{array}$ \\
\hline 2 & $\begin{array}{l}\text { Education: } \\
\begin{aligned} & \text { a. } \text { Non literate } \\
& \text { b. } \text { Primary } \\
& \text { c. } \text { High school } \\
& \text { d. } \text { Collegiate } \\
&\end{aligned}\end{array}$ & $\begin{array}{l}12 \\
1 \\
4 \\
0\end{array}$ & $\begin{array}{l}40 \\
3.3 \\
13 \\
0\end{array}$ & $\begin{array}{l}2 \\
6 \\
3 \\
0\end{array}$ & $\begin{array}{l}6.6 \\
20 \\
10 \\
0\end{array}$ & $\begin{array}{l}0 \\
0 \\
0 \\
2\end{array}$ & $\begin{array}{l}0 \\
0 \\
0 \\
6.6\end{array}$ & $\begin{array}{c}X^{2}=15.86 \\
D f=4 S \\
p>0.05\end{array}$ \\
\hline 3 & \begin{tabular}{cl}
\multicolumn{2}{l}{ Occupation: } \\
e. & House wife \\
f. & Employed \\
g. & Agriculture \\
h. & Others
\end{tabular} & $\begin{array}{l}12 \\
2 \\
3 \\
0\end{array}$ & $\begin{array}{l}40 \\
6.6 \\
10 \\
0\end{array}$ & $\begin{array}{l}7 \\
2 \\
3 \\
0\end{array}$ & $\begin{array}{l}23 \\
6.6 \\
10 \\
0\end{array}$ & $\begin{array}{l}0 \\
0 \\
0 \\
1\end{array}$ & $\begin{array}{l}0 \\
0 \\
0 \\
3.3\end{array}$ & $\begin{array}{c}X^{2}=4.4 \\
D f=4 N S \\
p>0.05\end{array}$ \\
\hline 4 & 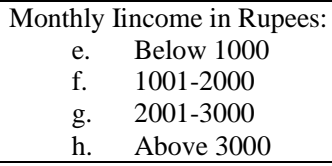 & $\begin{array}{l}7 \\
6 \\
3 \\
1 \\
\end{array}$ & $\begin{array}{l}23 \\
20 \\
10 \\
3.3 \\
\end{array}$ & $\begin{array}{l}5 \\
4 \\
2 \\
0 \\
\end{array}$ & $\begin{array}{l}16 \\
13 \\
6.6 \\
0 \\
\end{array}$ & $\begin{array}{l}1 \\
1 \\
0 \\
0 \\
\end{array}$ & $\begin{array}{l}3.3 \\
3.3 \\
0 \\
0 \\
\end{array}$ & $\begin{array}{c}\mathrm{X}^{2}=2.20 \\
\mathrm{Df}=6 \mathrm{NS} \\
\mathrm{p}>0.05\end{array}$ \\
\hline 5 & $\begin{array}{cl}\text { Religion: } & \\
\text { e. } & \text { Hindu } \\
\text { f. } & \text { Christian } \\
\text { g. } & \text { Muslim } \\
\text { h. } & \text { Others } \\
\end{array}$ & $\begin{array}{l}11 \\
5 \\
1 \\
0 \\
\end{array}$ & $\begin{array}{l}36 \\
16 \\
3.3 \\
0\end{array}$ & $\begin{array}{l}0 \\
6 \\
5 \\
0 \\
\end{array}$ & $\begin{array}{l}0 \\
20 \\
16 \\
0 \\
\end{array}$ & $\begin{array}{l}2 \\
0 \\
0 \\
0\end{array}$ & $\begin{array}{l}6.6 \\
0 \\
0 \\
0 \\
\end{array}$ & $\begin{array}{c}X^{2}=3.142 \\
D f=4 N S \\
p>0.05\end{array}$ \\
\hline 6 & $\begin{array}{cl}\text { Marital } & \text { Status: } \\
\text { e. } & \text { Single } \\
\text { f. } & \text { Married } \\
\text { g. } & \text { Widow } \\
\text { h. } & \text { Divorce } \\
\end{array}$ & $\begin{array}{l}1 \\
12 \\
4 \\
0 \\
\end{array}$ & $\begin{array}{l}3.3 \\
40 \\
13 \\
0 \\
\end{array}$ & $\begin{array}{l}0 \\
10 \\
1 \\
0 \\
\end{array}$ & $\begin{array}{l}0 \\
3.3 \\
3.3 \\
0 \\
\end{array}$ & $\begin{array}{l}0 \\
0 \\
2 \\
0 \\
\end{array}$ & $\begin{array}{l}0 \\
0 \\
6.6 \\
0 \\
\end{array}$ & $\begin{array}{c}X^{2}=2.37 \\
D f=6 N S \\
p>0.05\end{array}$ \\
\hline 7 & $\begin{aligned} & \text { Parity: } \\
& \text { e. } \text { Nullipara } \\
& \text { f. } \text { Primipara } \\
& \text { g. } \text { Multipara } \\
& \text { h. } \text { Grand multipara } \\
&\end{aligned}$ & $\begin{array}{l}0 \\
3 \\
12 \\
2 \\
\end{array}$ & $\begin{array}{l}0 \\
10 \\
40 \\
6.6\end{array}$ & $\begin{array}{l}2 \\
1 \\
3 \\
5\end{array}$ & $\begin{array}{l}6.6 \\
3.3 \\
10 \\
6\end{array}$ & $\begin{array}{l}0 \\
0 \\
1 \\
1\end{array}$ & $\begin{array}{l}0 \\
0 \\
3.3 \\
3.3 \\
\end{array}$ & $\begin{array}{c}X^{2}=9.72 \\
D f=6 N S \\
p>0.05\end{array}$ \\
\hline
\end{tabular}

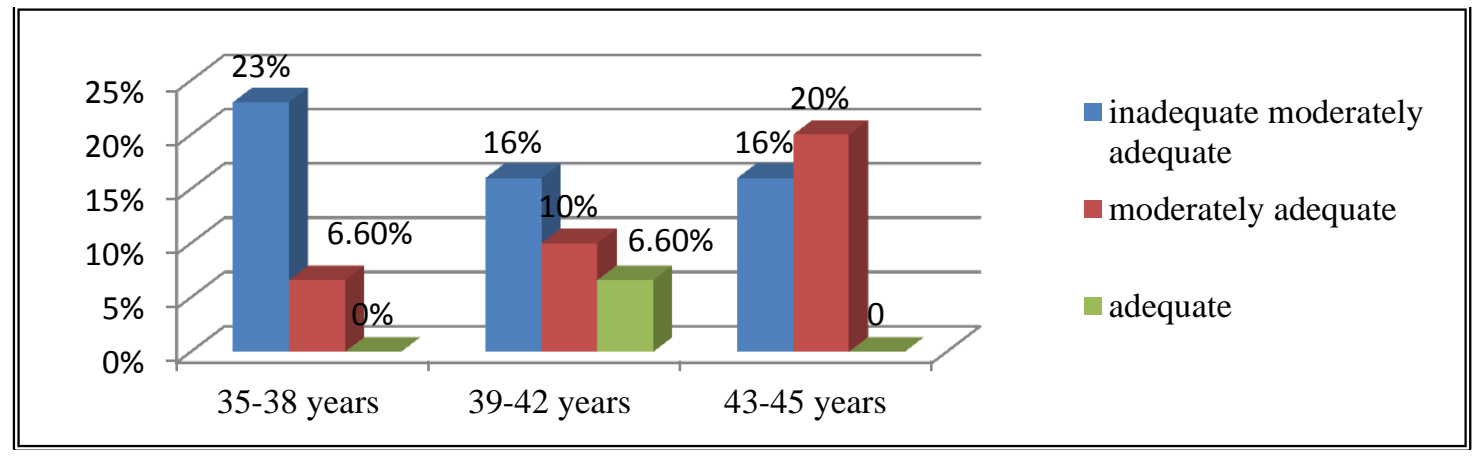

Figure: 2 Association between level of knowledge and age among premenopausal women

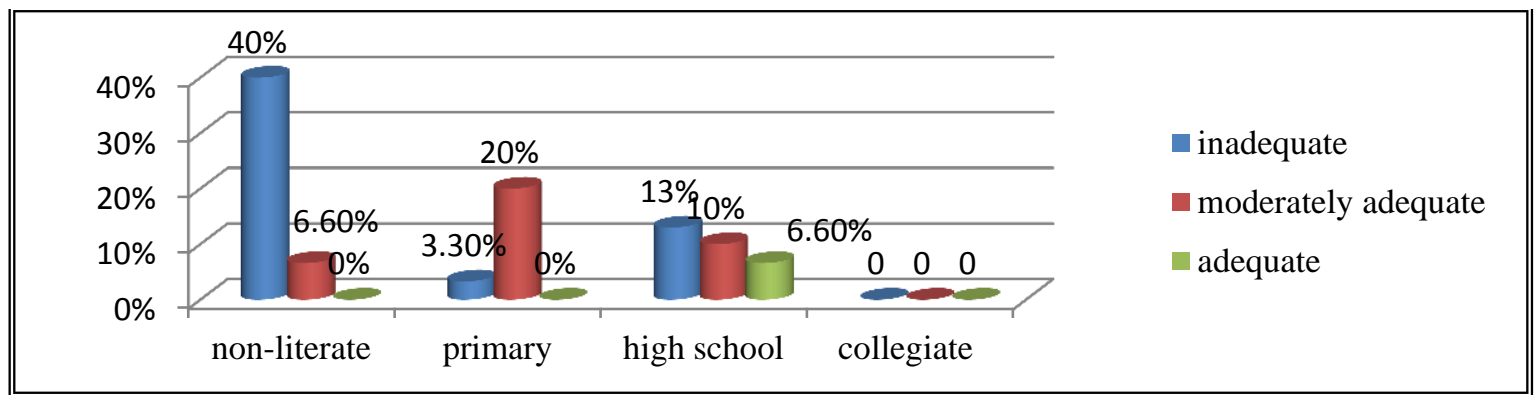

Figure: 3 Association between level of knowledge and educational status 


\section{Conclusion}

Health education is an integral part of Nursing practice so Nurses working in the community impart education to women in Menopausal period. Continuing Research and utilization of the findings in practice will make the public and health professional to understand more about the management of Menopausal symptoms.

\section{References}

[1]. Bachmann . G.A and Leiblum SR. 2004. The impact of Harmones on Menopausal sexuality, Medline.

[2]. Irene M.Boback and Margaret Duncan Jensen(1993). "Maternity and gynaecological Care". USA, Mosby Publications.

[3]. Thayamalar G. Gomala(2004). "Menopause Special Guide for Ladies crossing above 40 years". Aval Vigaden Publications.

[4]. Shoenfeld H.(1999). When are Menopausal Symptoms Becomes Psychiatric”. Medline.

[5]. Thomson J. and Oswald I. (1997). "Effect of Oestrogen on the sleep, Mood and Anxiety of Menopausal women". Medline.

[6]. P.S.S. Sundar Rao and J. Richard (2003). "An Introduction to Biostatics". New Delhi. Prentice Hall of India Private Limited. 